\title{
STRONGLY ADDITIVE TRANSFORMATIONS AND INTEGRAL REPRESENTATIONS WITH MEASURES OF NONLINEAR OPERATORS
}

\author{
BY JƯRGEN BATT
}

\author{
Communicated by P. D. Lax, October 25, 1971
}

In the development of the representation theory for linear transformations on function spaces by integrals, historically three different stages can be distinguished: in stage (I) one considers scalar valued transformations (functionals) on scalar valued functions, in stage (II) the transformations (stage (IIA)) or the functions (stage (IIB)) assume values in a topological vector space, and in stage (III) one studies the case of (vector valued) transformations on a space of vector valued functions. If we restrict ourselves to Banach spaces, as for the space of continuous functions on a compact Hausdorff space, stage (I) is marked by the theorems of F. Riesz [19] and S. Kakutani [14], (IIA) by the results of R. G. Bartle, N. Dunford and J. Schwartz [1], (IIB) by the investigations of S. Bochner and A. E. Taylor [6] and I. Singer [20], (III) by the results of [5], of N. Dinculeanu [8], and C. Foias and I. Singer [10]. In a similar way, the history of representation theory for linear maps on $L^{p}$-spaces could be retraced. Recently however, most interesting features have been added to the theory by the introduction and consideration of a wide class of nonlinear transformations, for example those $T$, for which $T\left(f_{1}+f_{2}\right)=T f_{1}+T f_{2}$ for all pairs of functions $f_{1}, f_{2}$ for which the intersection of the sets $\left\{t: f_{i}(t) \neq 0\right\}, i=1,2$, is empty or has measure zero. The theory of these "additive" transformations on the $L^{p}$-spaces was begun with results of L. Drewnowski and W. Orlicz [9] and V. Mizel and K. Sundaresan [15], [16], [17] (stage (I)) and rather quickly reached the stages (II) and (III) by a subsequent paper of V. Mizel and K. Sundaresan [18]. As for the nonlinear transformations on spaces of continuous functions it seems evident that the additivity alone is not sufficient to build up a satisfactory representation theory. Stage (I) of their theory began with the research of R. V. Chacon and N. A. Friedman [7] and of N. A. Friedman and M. Katz [11], [12] and with their introduction of a generalized additivity property (called "strong additivity" below). Since these papers appeared the writer of this note worked out the theory in its stage (III) following the line of his earlier work in linear theory and presented his results at a conference on vector measures at the University of Utah in April 1971 not being aware of the fact that the theory had

AMS 1970 subject classifications. Primary 46G10, 47H99.

Key words and phrases. Integral representation, additive transformation, weakly compact transformation, vector measure. 
already entered its stage (IIA) by results of N. A. Friedman and A. E. Tong [13] which came to his knowledge after their publication in May 1971. The purpose of this note is to announce the following further results, the proofs of which will be published elsewhere.

1. The integral. Let $E$ and $F$ be Banach spaces and let $E_{\alpha}:=\{x \in E,\|x\|$ $\leqq \alpha\}, \alpha>0$. Consider the linear space $M(E, F)$ of all mappings $U: E \rightarrow F$ with the following properties:

(i) $U 0=0$,

(ii) $\left\|U_{\alpha}\right\|:=\sup _{x \in E_{\alpha}}\|U x\|<\infty\left(U_{\alpha}\right.$ is the restriction of $U$ on $\left.E_{\alpha}\right)$,

(iii) $D_{\delta}^{\alpha} U:=\sup _{x, x^{\prime} \in E_{x} ;\left\|x-x^{\prime}\right\| \leqq \delta}\left\|U x-U x^{\prime}\right\|($ defined for $\alpha, \delta>0)$ tends to zero for $\delta \rightarrow 0$ (and fixed $\alpha>0$ ).

(Property (i) is not essential and introduced for convenience.) We consider the linear space $M_{\alpha}(E, F)=\left\{U_{\alpha}: U \in M(E, F)\right\}$ normed by $\|\cdot\|: U_{\alpha}$ $\rightarrow\left\|U_{\alpha}\right\|$.

Now let $\mathscr{R}$ be a ring of subsets of an abstract set $S$ and let $S \in \mathscr{R}$. We define an integral for certain additive functions $U: \mathscr{R} \rightarrow M(E, F)$. For $g$ in the space $\mathscr{E}_{E}(\mathscr{R})$ of all $E$-valued, $\mathscr{R}$-simple functions we may and shall define

$$
\int g(t) d U(t)=\sum_{j} U\left(B_{j}\right) x_{j}
$$

where $g=\sum_{j} x_{j} \chi_{B_{j}}$ is a representation of $g$ by a finite linear combination of functions $x_{j} \chi_{B_{j}}$, with $x_{j} \in E, B_{j} \in \mathscr{R}$ and $\left\{B_{1}, \ldots, B_{r}\right\}$ being a disjoint partition of $S$ (such partitions will be denoted by $\pi$ in the sequel). Assume now that $U$ is "of bounded semivariation" on $S$. By this we mean that

and

$$
s v_{\alpha}(U, S):=\sup _{\substack{\pi \\ x_{j} E_{\alpha}}}\left\|\sum_{j} U\left(B_{j}\right) x_{j}\right\|<\infty
$$

$$
s v_{\delta}^{\alpha}(U, S):=\sup _{x_{j}, x_{j}^{\prime} \in E_{\alpha} ;\left\|x_{j}-x_{j}^{\prime}\right\| \leqq \delta}\left\|\sum_{j}\left(U\left(B_{j}\right) x_{j}-U\left(B_{j}\right) x_{j}^{\prime}\right)\right\| \rightarrow 0 \quad(\delta \rightarrow 0) .
$$

Then we may and shall define for all $g$ in the space $\mathscr{M}_{E}(\mathscr{R})$ of all $E$-valued, $\mathscr{R}$-totally measurable functions

$$
\int g(t) d U(t)=\lim _{n \rightarrow \infty} \int g_{n}(t) d U(t),
$$

where $\left\{g_{n}\right\}$ is a sequence in $\mathscr{E}_{E}(\mathscr{R})$ tending to $g$ uniformly on $S$. For all $g, g_{1}$, $g_{2} \in \mathscr{M}_{E}(\mathscr{R})$ with $\left\{t: g_{1}(t) \neq 0\right\} \cap\left\{t: g_{2}(t) \neq 0\right\}=\varnothing$ we then have

$$
\int\left(g+g_{1}+g_{2}\right) d U=\int\left(g+g_{1}\right) d U+\int\left(g+g_{2}\right) d U-\int g d U .
$$


2. The representation theorem. We consider a compact Hausdorff space $S$ and denote by $C_{E}(S)$ the space of all continuous functions defined on $S$ with values in $E$, endowed with the uniform norm. $\mathscr{B}$ is the $\sigma$-ring of the Borel sets in $S$.

THEOREM 1. For any $T \in M\left(C_{E}(S), F\right)$, which is "strongly additive," that is for which

$$
T\left(f+f_{1}+f_{2}\right)=T\left(f+f_{1}\right)+T\left(f+f_{2}\right)-T f
$$

for all $f, f_{1}, f_{2} \in C_{E}(S)$ with supp $f_{1} \cap \operatorname{supp} f_{2}=\varnothing$, there exists a unique finitely additive function $U: \mathscr{B} \rightarrow M\left(E, F^{\prime \prime}\right)$ of bounded semivariation on $S$, such that $\mu_{y^{\prime} \alpha}: \mathscr{B} \rightarrow M_{\alpha}(E, C)$ is regular for all $y^{\prime} \in F^{\prime}$ and $\alpha>0$, one has

$$
T f=\int f d U, \quad f \in C_{E}(S),
$$

and $\left\|T_{\alpha}\right\|=s v_{\alpha}(U, S), \quad D_{\delta}^{\alpha} T=s v_{\delta}^{\alpha}(U, S), \alpha, \delta>0$. The measures $\mu_{y^{\prime}}: \mathscr{B}$ $\rightarrow M(E, C)$ are defined by $\mu_{y^{\prime}}(B) x=\left\langle U(B) x, y^{\prime}\right\rangle$ for all $B \in \mathscr{B}, x \in E$.

The proof is based on a representation theorem for strongly additive functionals. The representation of these functionals obtained in [12] for the special case $E=\boldsymbol{R}$ can easily be derived from this result using the existence of a lifting for the measure

$$
\sum_{n=1}^{\infty} \frac{1}{2^{n}} \frac{v\left(\mu_{n}\right)}{1+v\left(\mu_{n}, S\right)}
$$

where $\mu$ is the representing measure and $v\left(\mu_{n}\right)$ the variation of $\mu_{n}: \mathscr{B}$ $\rightarrow M_{n}(E, C)$, and the Radon-Nikodým theorem. This argument furnishes at the same time a kernel representation for functionals in case $E$ is an arbitrary Banach space.

3. Consequences of the representation theorem. The assumptions of Theorem 1 will be made throughout.

THEOREM 2. If no subspace of $F$ is isomorphic to $c_{0}$ or if $T$ is weakly compact then the measures $v\left(\mu_{y^{\prime} \alpha}\right)$ are $\sigma$-additive uniformly for $\left\|y^{\prime}\right\| \leqq 1$ and each $\alpha>0$.

This last property has been extensively studied in [4] for linear operators $T$. In particular, it implies that $U: \mathscr{B} \rightarrow M(E, F)$ and that the functions $U_{\alpha}: \mathscr{B} \rightarrow M_{\alpha}(E, F)$ are $\sigma$-additive in the norm of $M_{\alpha}(E, F)$. Under the assumptions of Theorem 2 it follows in the case $E=C$ that $T$ takes weak Cauchy sequences in strong Cauchy sequences.

4. Special results related to the weak compactness of $T$. In the linear case 
it is known that if no subspace of $F$ is isomorphic to $c_{0}$ and if one of the following conditions holds :

(i) $E$ is reflexive,

(ii) $E^{\prime \prime}$ is separable and all $U(B), B \in \mathscr{B}$ are weakly compact, then $T$ is weakly compact (see [3]). We have the following unifying result valid even for strongly additive $T \in M\left(C_{E}(S), F\right)$.

THEOREM 3. If the measures $v\left(\mu_{y^{\prime} \alpha}\right)$ are $\sigma$-additive uniformly for $\left\|y^{\prime}\right\| \leqq 1$ and each $\alpha>0$, if there exists a linear space $E^{\beta} \subset M(E, C)$ such that $\mu_{y^{\prime}}: \mathscr{B} \rightarrow E^{\beta}, y^{\prime} \in F^{\prime}$ and the closures $\bar{E}_{\alpha}^{\beta}$ of $E_{\alpha}^{\beta}$ in $M_{\alpha}(E, C)$ together with the duals $\bar{E}_{\alpha}^{\beta^{\prime}}$ possess the Radon-Nikodým property, $\alpha>0$, and if all $U(B), B \in \mathscr{B}$, are weakly compact, then $T$ is weakly compact.

The crucial point in the proof is the use of an extension of the Gantmacher theorem to nonlinear mappings as established in [2]. Let us also remark that there exist counterexamples for the conclusion being false in cases that for $E^{\beta}=\operatorname{span}\left\{\mu_{y^{\prime}}(B), B \in \mathscr{B}, y^{\prime} \in F^{\prime}\right\}$ either the $E_{\alpha}^{\beta}$ or the $E_{\alpha}^{\beta^{\prime}}$ fail to have the Radon-Nikodym property (with the other conditions being satisfied).

Using a criterion for the weak compactness of subsets of spaces of regular $\sigma$-additive measures with values in the space of continuous functions over the unit disk in the complex plane we are able to prove, for $E=C$,

THEOREM 4. If no subspace of $F$ is isomorphic to $c_{0}$ then every strongly additive transformation $T \in M(C(S), F)$ is weakly compact.

\section{REFERENCES}

1. R. G. Bartle, N. Dunford and J. Schwartz, Weak compactness and vector measures, Canad. J. Math. 7 (1955), 289-305. MR 16, 1123.

2. J. Batt, Nonlinear compact mappings and their adjoints, Math. Ann. 189 (1970), 5-25.

3. - Applications of the Orlicz-Pettis theorem to operator valued measures and compact and weakly compact linear transformations on the space of continuous functions, Rev. Roumaine Math. Pures Appl. 14 (1969), 907-935.

4. J. Batt and E. Jeffrey Berg, Linear bounded transformations on the space of continuous functions, J. Functional Analysis 4 (1969), 215-239. MR 40 \#1798.

5. J. Batt und $\mathrm{H}$. König, Darstellung linearer Transformationen durch vektorwertige Riemann-Stieltjes-Integrale, Arch. Math. 10 (1959), 273-287. MR 21 \# 7423.

6. S. Bochner and A. E. Taylor, Linear functionals on certain spaces of abstractly-valued functions, Ann. of Math. (2) 39 (1938), 913-944.

7. R. V. Chacon and N. Friedman, Additive functionals, Arch. Rational Mech. Anal. 18 (1965), 230-240. MR 30 \# 2329.

8. N. Dinculeanu, Sur la représentation intégrale de certaines opérations linéaires. III, Proc. Amer. Math. Soc. 10 (1959), 59-68. MR 21 \# 2909.

9. L. Drewnowski and W. Orlicz, On orthogonally additive functionals, Bull. Acad. Polon. Sci. Sér. Sci. Math. Astronom. Phys. 16 (1968), 883-888. MR 39 \# 6069.

10. C. Foias and I. Singer, Some remarks on the representation of linear operators in spaces of vector-valued continuous functions, Rev. Roumaine Math. Pures Appl. 5 (1960), 729-752. MR 24 \# A1618.

11. N. Friedman and M. Katz, A representation theorem for additive functionals, Arch. Rational Mech. Anal. 21 (1966), 49-57. MR 33 \# 245. 
12. - On additive functionals, Proc. Amer. Math. Soc. 21 (1969), 557-561. MR 39 \#4653.

13. N. Friedman and A. E. Tong, On additive operators, Canad. J. Math. 23 (1971), 468-480.

14. S. Kakutani, Concrete representation of abstract $(M)$-spaces ( $A$ characterization of the space of continuous functions), Ann. of Math. (2) 42 (1941), 994-1024. MR 3, 7; 8, 205.

15. V. J. Mizel and K. Sundaresan, Representation of additive and biadditive functionals,

Arch. Rational Mech. Anal. 30 (1968), 102-126. MR 37 \#3341.

16. V. J. Mizel, Representation of nonlinear transformations on $L^{p}$ spaces, Bull. Amer. Math. Soc. 75 (1969), 164-168. MR 38 \# 4983.

17. Characterization of non-linear transformations possessing kernels, Canad. J. Math. 22 (1970), 449-471. MR 41 \# 7495.

18. V. J. Mizel and K. Sundaresan, Representation of vector valued nonlinear functions, Trans. Amer. Math. Soc. 159 (1971), 344-379.

19. F. Riesz, Sur les opérations fonctionelles linéaires, C.R. Acad. Sci. Paris 149 (1909), 974-977.

20. I. Singer, Linear functionals on the space of continuous mappings of a compact Hausdorff space into a Banach space, Rev. Roumaine Math. Pures Appl. 2 (1957), 301-315. (Russian) MR 20 \# 3445.

Mathematisches Institut, Universitat MünChen, 8 MünChen 13, Schellingstrasse 2-8, Federal Republic of Germany 